Reprod. Nutr. Dévelop., 1988, 28 (1), 157-158.

\title{
Dégradabilité in sacco de la matière sèche et des constituants pariétaux dans le rumen. Effet de l'apport de matières grasses dans la ration de la vache laitière.
}

M. DOREAU, Brigitte MICHALET-DOREAU (*)

Laboratoire de la Lactation

${ }^{*}{ }^{*}$ Laboratoire des Aliments

I.N.R.A., Theix, 63122 Ceyrat, France.

Summary. The effect of fat in dairy rations was studied by in sacco dry matter and fiber disappearance in the rumen. Fat supplementation did not modify the non degradable fraction, but the degradation rate constant for the slowly degradable fraction decreased. This decreases, was marked for dry matter as for fiber.

L'addition de matières grasses dans la ration des ruminants entraîne souvent une diminution de la digestibilité de la ration, principalement en raison de la réduction de la digestion des constituants pariétaux dans le rumen (Devendra et Lewis, 1974). La digestion de ces parois peut être appréciée à partir de la mesure de la dégradabilité in sacco (Demarquilly et Chenost, 1969). L'objet de ce travail est de quantifier les effets de l'addition de matières grasses dans la ration sur la cinétique de dégradation de la matière sèche $(\mathrm{MS})$ et des constituants pariétaux dans le rumen.

Matériel et méthodes. Deux essais ont été réalisés, chacun sur 3 vaches laitières munies de fistules du rumen, en milieu de lactation, avec des régimes composés de foin $(60 \%)$ et d'aliment concentré $(40 \%)$ contenant soit du tourteau de colza soit des graines de colza (essai 1), ou des régimes composés d'ensilage de maïs $(60 \%)$ et de l'aliment concentré $(40 \%)$ contenant ou non de la graisse d'os de bovin (essai 2). Les pourcentages d'acides gras dans la MS étaient respectivement 2,5 et 8,4 (essai 1 ) ; 2,5 et 9,7 (essai 2). Les régimes contenaient environ $1,1 \%$ de calcium. Chaque période expérimentale durait 5 semaines dont 1 (la dernière) de mesure. Les rations ont été distribuées en 2 fois par jour et en quantité limitée, ajustée en fonction de la production laitière.

Des sachets (taille de pores : $20-80 \mu \mathrm{m}$ ), contenant $3 \mathrm{~g}$ du foin (essai 1) ou de l'ensilage de maïs sans les grains (cannes + feuilles) (essai 2 ) distribués dans la ration, ont été placés pendant $3,6,12,24$ et $48 \mathrm{~h}$ dans le rumen. A chaque durée d'incubation correspondaient 6 mesures ( 3 animaux $\times 2$ répétitions). Les dosages des parois totales (NDF) et de la lignocellulose (ADF) sur les résidus ont été réalisés après regroupement des sachets correspondant à une même durée d'incubation et à un même régime.

Résultats et discussion. La dégradation de la MS est légèrement plus faible avec les régimes enrichis en matières grasses. Cette diminution apparaît à partir de $12 \mathrm{~h}$ d'incubation dans le rumen, mais les variations restent non significatives quelles que soient la durée considérée et la nature de la source de matières grasses ou de la ration (tabl. 1).

Reproduction, Nutrition, Développement, $n^{\circ} 1-88 .-11$ 
TABL. 1. - Effet de l'apport de matières grasses (MG) sur la cinétique de dégradation dans le rumen de la matière sèche et des constituants pariétaux de foin (essai 1) et d'ensilage de mäs (essai 2).

\begin{tabular}{|c|c|c|c|c|c|c|c|c|c|c|c|}
\hline \multirow{2}{*}{\multicolumn{2}{|c|}{ Durée d'incubation (h) }} & \multicolumn{5}{|c|}{$\begin{array}{c}\text { Essai } 1 \\
\text { graine de colza } \\
\end{array}$} & \multicolumn{5}{|c|}{$\begin{array}{c}\text { Essai } 2 \\
\text { graisse d'os }\end{array}$} \\
\hline & & \multirow{2}{*}{$\begin{array}{c}3 \\
48,1 \\
47,5\end{array}$} & \multirow{2}{*}{$\begin{array}{c}6 \\
53,7 \\
52,0\end{array}$} & \multirow{2}{*}{$\begin{array}{c}12 \\
65,6 \\
59,9\end{array}$} & \multirow{2}{*}{$\begin{array}{c}24 \\
72,1 \\
70,2\end{array}$} & \multirow{2}{*}{$\begin{array}{c}48 \\
82,1 \\
79,1\end{array}$} & \multirow{2}{*}{$\begin{array}{c}3 \\
46,9 \\
46,9\end{array}$} & \multirow{2}{*}{$\begin{array}{c}6 \\
50,5 \\
49,3\end{array}$} & \multirow{2}{*}{$\begin{array}{c}12 \\
56,3 \\
53,6\end{array}$} & \multirow{2}{*}{$\begin{array}{c}24 \\
66,3 \\
62,3\end{array}$} & \multirow{2}{*}{$\begin{array}{c}48 \\
72,7 \\
70,7\end{array}$} \\
\hline MS & $\begin{array}{l}\text { témoin } \\
+\mathrm{MG}\end{array}$ & & & & & & & & & & \\
\hline NDF & $\begin{array}{l}\text { témoin } \\
+M G\end{array}$ & $\begin{array}{l}22,2 \\
21,4\end{array}$ & $\begin{array}{l}29,7 \\
27,5\end{array}$ & $\begin{array}{l}47,2 \\
38,4\end{array}$ & $\begin{array}{l}58,8 \\
51,6\end{array}$ & $\begin{array}{l}73,6 \\
67,4\end{array}$ & $\begin{array}{l}20,3 \\
20,0\end{array}$ & $\begin{array}{l}25,2 \\
24,2\end{array}$ & $\begin{array}{l}31,4 \\
29,2\end{array}$ & $\begin{array}{l}48,5 \\
41,4\end{array}$ & $\begin{array}{l}58,8 \\
55,1\end{array}$ \\
\hline ADF & $\begin{array}{l}\text { témoin } \\
+M G\end{array}$ & $\begin{array}{l}8,5 \\
5,7\end{array}$ & $\begin{array}{l}18,7 \\
13,5\end{array}$ & $\begin{array}{l}38,8 \\
26,0\end{array}$ & $\begin{array}{l}50,7 \\
42,3\end{array}$ & $\begin{array}{l}70,2 \\
63,0\end{array}$ & $\begin{array}{l}14,3 \\
14,9\end{array}$ & $\begin{array}{l}17,6 \\
17,7\end{array}$ & $\begin{array}{l}26,8 \\
20,7\end{array}$ & $\begin{array}{l}41,4 \\
34,3\end{array}$ & $\begin{array}{l}52,7 \\
49,1\end{array}$ \\
\hline NDF-ADF & $\begin{array}{l}\text { témoin } \\
+M G\end{array}$ & $\begin{array}{l}37,4 \\
38,9\end{array}$ & $\begin{array}{l}41,9 \\
43,1\end{array}$ & $\begin{array}{l}56,5 \\
52,1\end{array}$ & $\begin{array}{l}67,9 \\
60,8\end{array}$ & $\begin{array}{l}77,5 \\
72,4\end{array}$ & $\begin{array}{l}26,9 \\
25,6\end{array}$ & $\begin{array}{l}33,5 \\
31,1\end{array}$ & $\begin{array}{l}41,1 \\
38,5\end{array}$ & $\begin{array}{l}56,3 \\
49,2\end{array}$ & $\begin{array}{l}65,4 \\
61,7\end{array}$ \\
\hline
\end{tabular}

Pour faciliter l'interprétation des cinétiques précédentes, nous avons réalisé une modélisation selon une fonction exponentielle du temps (Sauvant et al., 1985 ; Robinson et al., 1986), soit : $\mathrm{D}(\mathrm{t})=\mathrm{Dr}+\mathrm{DI}\left(1-\mathrm{e}^{-\mathrm{k}(\mathrm{t})}\right.$. Ce modèle du $1_{\text {er }}$ ordre différencie 3 fractions : la première très rapidement dégradable $\mathrm{Dr}$, la deuxième lentement dégradable $\mathrm{DI}$ à un taux de dégradation $\mathrm{kl}$ et la troisième non dégradable $(100-\mathrm{Dr}-\mathrm{DI})$. L'importance relative de ces 3 fractions n'est pas modifiée par l'apport de matières grasses dans la ration, mais le taux de dégradation $\mathrm{kI}$ de la MS diminue significativement de $34 \%$ avec la graine de colza et de $45 \%$ avec la graisse d'os. Le recours au modèle permet ainsi de mieux mettre en évidence et comprendre les différences entre régimes.

L'action des matières grasses sur la dégradation des constituants pariétaux (NDF, ADF et NDF-ADF) est du même type : seul le taux de dégradation est diminué, respectivement de 34,29 et $30 \%$ dans l'essai 1 , et de 55,52 et $39 \%$ dans l'essai 2.

Ces équations permettent de simuler les quantités de MS dégradées dans le rumen par la prise en compte du taux de renouvellement de la MS dans le rumen, qui était en moyenne de 0,038 et $0,040 \mathrm{~h}^{-1}$ dans l'essai 1, respectivement pour les régimes témoin et graine de colza (Doreau et al., non publié) et estimé à 0,040 pour les deux régimes de l'essai 2 . La dégradabilité théorique de la MS diminue significativement avec l'apport de matières grasses, passant de 70 à $64 \%$ pour l'essai 1 et de 61 à $58 \%$ pour l'essai 2.

Les matières grasses semblent agir uniquement en réduisant le taux de disparition $\mathrm{kl}$ de la fraction lentement dégradable de la MS. Cette réduction pourrait être à l'origine de la diminution de la quantité de MS digérée dans le rumen avec les régimes enrichis en matières grasses (Sutton et al., 1983).

Demarquilly C., Chenost M., 1969. Ann. Zootech., 18, 419-436.

Devendra P., Lewis D., 1974. Anim. Prod., 19, 67-76.

Robinson P. H., Fadel J. G., Tamminga S., 1986. Anim. Feed Sci. Technol., 15, 249-271.

Sauvant D., Bertrand D., Giger S., 1985. Anim. Feed Sci. Techol., 13, 7-23.

Sutton J. D., Knight R., McAllan A. B., Smith R. H., 1983. Br. J. Nutr., 49, 419-432. 